\title{
Genetics of Dizygotic Twinning: A Feasibility Study for a Biobank
}

\author{
Chantal Hoekstra,' Piet Meijer, ${ }^{2}$ Cornelis Kluft, ${ }^{2}$ Peter Heutink, ${ }^{1,3}$ Guus Smit, ${ }^{4}$ Eco de Geus,' Jan H. Smit, \\ Angelique van Bruggen,' Grant W. Montgomery, ${ }^{6}$ and Dorret I. Boomsma ${ }^{1,3}$ \\ 'Department of Biological Psychology,Vrije Universiteit, Van der Boechorststraat I, I08I BT Amsterdam, the Netherlands \\ ${ }^{2}$ Gaubius Laboratory, TNO-PG Leiden. Zernikedreef 9, 2333 CK Leiden, the Netherlands \\ ${ }^{3}$ VUmc Section Medical Genomics Amsterdam, De Boelelaan 1085, 108I HV Amsterdam, the Netherlands \\ ${ }^{4}$ Department of Molecular and Cellular Neurobiology Amsterdam, Faculty of Earth and Life Sciences, Vrije Universiteit, De Boelelaan I085, \\ I08I HV Amsterdam, the Netherlands \\ ${ }^{5}$ Deptartment of Psychiatry Amsterdam, Valeriusplein 9, 1075 BG Amsterdam, the Netherlands \\ ${ }^{6}$ Genetic Epidemiology Laboratory, Queensland Institute of Medical Research, 300 Herston Road, Brisbane, Australia
}

\begin{abstract}
Tionson o locate the genes that make a substantial contribution to variation in natural dizygotic twinning in humans, large-scale studies are needed. New studies should not stop at DNA genotyping, but collect material that allow gene-expression analysis, transcriptomics, proteomics and endocrinology. In this article we describe a pilot study to examine the feasibility, effectiveness and logistics of large-scale nationwide sample collection in Dutch families in which two or more sisters have given birth to spontaneous dizygotic twins. Pedigree data and addresses from family members of proband mothers were collected by telephone. Blood and urine samples were collected during a home visit, and handled in the afternoon. All participants were bled between 7 a.m. and 10 a.m. after overnight fasting. Blood samples of fertile women with a natural cycle were collected on the second, third or fourth day of their menstrual cycle. The effects of transportation and storage on blood quality, lipids, RNA with and without challenge, lymphocytes and other parameters were examined. Genomic DNA was isolated from blood and cells were immortalized using Epstein-Barr virus. In $78.6 \%$ of the women with a natural cycle blood samples were collected on the second, third or fourth day of the menstrual cycle. This percentage is likely to increase with the more dense geographical distribution of participants in the larger population. We conclude that the pilot study demonstrated the feasibility of this protocol to collect good quality of plasma, DNA, RNA and lymphocyte samples by home visits.
\end{abstract}

It has been firmly established that variation in dizygotic (DZ) twinning has a genetic component (Bulmer, 1970). Family studies found significantly higher frequencies of DZ twins in female relatives of DZ probands compared with monozygotic (MZ) probands. Both autosomal monogenic dominant and recessive models of inheritance have been described (Lewis et al., 1996;
Meulemans et al., 1996). However, the genetic architecture of twinning in humans remains unclear.

There are good animal models of twinning in mammals, especially in sheep. An important question is whether natural variation in human twinning is controlled by some of the same mechanisms as have been identified in sheep, or through other pathways. For example, mutations in three members (BMP15, BMPR1B and GDF9) of the intraovarian transforming growth factor beta (TGFb) signaling pathway increase the frequency of twins and higher-order multiples, in sheep (Galloway et al., 2000; Hanrahan et al., 2004; Wilson et al., 2001). Mutations in BMP15 and GDF9 are associated with both increased ovulation rate and sterility in Cambridge and Belclare sheep (Hanrahan et al., 2004).

One reason for the lack of success so far to identify the genetic contribution to human DZ twinning may be the small sample size of many studies. We are therefore recruiting a large sample of sister pairs who have both given birth to spontaneous DZ twins. Affected sister pairs, their parents and other informative family members will be recruited in the Netherlands, in Australia and New Zealand. In the Netherlands, 500 families will be identified through the Netherlands Twin Registry (NTR) and in Australia and New Zealand, 500 families will be recruited through appeals in the media. The proposed study aims to extend our previous studies of the endocrinology, epidemiology and molecular genetics of twinning in humans (Boomsma et al., 1992; Lambalk et al., 1998; Martin et al., 1984; Martin et al., 1991; Montgomery et al., 2003).

The collection of biological material other than DNA samples that enables the study of the endocrinol-

Received 25 June, 2004; accepted 9 September, 2004.

Address for correspondence: Chantal Hoekstra, Department of Biological Psychology, Vrije Universiteit, Van der Boechorstraat 1, 1081 BT Amsterdam, the Netherlands. E-mail: C.Hoekstra@psy.vu.nl 
ogy of twinning, the identification of candidate genes through expression studies and possibly the characterization of affected women through system biology approaches requires the establishment of large biobanks. Biobanks can be defined as stored collections of genetic samples and biological materials from participants coming from selected groups or from random populations. Biological materials include genomic DNA, RNA, lymphocytes serum and/or plasma which enables investigators to perform long-term genomic, transcriptomic or proteomic studies (Austin et al., 2003; Kaiser, 2002; Nederhand et al., 2003). At the moment, characterization of individuals and families, using these techniques, including large-scale genotyping of microsatellites and SNPs, represents a major cost. Therefore, the aim is to collect multiple phenotypes in such studies, so that linkage and association analysis can be conducted for multiple phenotypes within the same families. To be cost-effective and to minimize the future burden for participants, a broad range of biomaterials should be collected in a single visit that allows future study of multiple (disease) traits and endophenotypes. Ideally, the multiple phenotypes reflect variation in physiological parameters, which are related to affection status, but with appropriate sample collection, large-scale studies will also provide a valuable resource for many traits that can be assessed in plasma or serum. Some of these traits require standardized protocols. For example, to assess lipid levels, glucose and insulin participants ideally are bled after overnight fasting and at a fixed time of the day. For other parameters, following a certain circadian pattern (such as cortisol) participants need to be bled at a fixed time of the day. To study the endocrinology of twinning, blood samples in fertile women need to be collected around the time of follicle recruitment.

In this paper, we describe a pilot study to examine the feasibility, effectiveness and logistics of large-scale nationwide biomaterials collection in the Netherlands to locate genes responsible for DZ twinning. We first describe the procedure for recruitment of families of affected sister pairs and next of phenotype and biological sample collection. The logistics of transport and blood handling and the procedures for blood handling and results of RNA isolation are described.

\section{$\overline{\text { Materials and Methods }}$ Participants}

The study was approved by the Central Ethics Committee on Research Involving Human Subjects of the Vrije Universiteit (Amsterdam). We approached 24 mothers of dizygotic twins (MODZT) registered with the Netherlands Twin Registry (NTR). These mothers reported in survey studies of the NTR having one or more sisters with a twin or multiple births. Most of them had responded to an article in the yearly newsletter of the NTR about this study.

The proband mother was contacted by letter, followed by a telephone call to collect pedigree data and to obtain permission to approach her sister, her parents and/or other siblings. All family members were initially informed about the study by the proband mother and after giving their permission to be contacted, were sent a letter and information brochure about the study. To be eligible for the study, two or more sisters had to have given birth to spontaneous dizygotic twins. If one or both parents were unavailable or deceased, other siblings were asked for their participation.

To assess hormone levels at the time of follicle recruitment in fertile women, blood and urine samples of women with a natural cycle were taken on the second, third or fourth day of the menstrual cycle. In women who used oral contraceptives (OC) blood and urine samples were taken in the week the women did not take OC pills. All of these women used combination OCs. Menopausal women, fathers and brothers did not have to meet these criteria. All participants gave written informed consent prior to participation in the study.

\section{Interview}

Pedigree data about DZ twinning and fertility were obtained from a telephone interview with the proband mother and her sister. The interview time was between 20 and 30 minutes. The interview for MODZT consisted of five parts. Part A contained 20 items regarding age, birth weight, parity and mother's judgment of zygosity of the twins. In part B, 11 items about birth history, use of hormone treatment or IVF in the conception of the twins and in other conceptions were asked. Part $\mathrm{C}$ asked about family history of twinning and fertility. Pedigree data were obtained for the family of the proband mother, for her mother's family, her father's family and for the family of her husband. Part D contained 19 items regarding health and medical history. Data were collected on height, weight (before, during and after the twin pregnancy), menstrual cycle, menopause (of proband and her mother), use of contraception and health problems. The last part of the questionnaire, part E, contained four items about ancestry of the parents and grandparents of the MODZT.

\section{Blood Sampling}

Venous blood samples and 'morning' urine samples were taken from the MODZT, their parents and/or additional siblings between $7.00 \mathrm{a} . \mathrm{m}$. and $10.00 \mathrm{a}$.m. and after overnight fasting. A total of seven blood tubes was collected from all participants for DNA, lymphocytes, RNA (basal and after dexamethasone challenge) and plasma. The order of sample draw was $2 \times 9 \mathrm{ml}$ EDTA, $3 \times 9 \mathrm{ml}$ Heparin, $1 \times 4.5 \mathrm{ml}$ citrate and $1 \times 2 \mathrm{ml}$ EDTA. For collection, we used a safetylock butterfly needle. With this system, tubes can be quickly and hygienically changed. To prevent clotting all tubes were inverted gently 8-10 times immediately after collection.

For DNA isolation and EDTA plasma, blood was collected in $2 \times 9 \mathrm{ml}$ EDTA anticoagulant tubes. The tubes were stored in melting ice $\left(0-2^{\circ} \mathrm{C}\right)$ during trans- 
Table 1

Overview of Traits Assessed in Blood/Plasma in all Participants

\begin{tabular}{lccccc}
\hline Blood variable & & $N$ & Mean & $S D$ & Range \\
\hline HbA1c & HbA1c (\%) & 69 & 5.6 & .44 & $4.5-7.1$ \\
CRP & $\mathrm{CRP}(\mathrm{mg} / \mathrm{L})$ & 73 & 5.45 & 12.10 & $.14-83.80$ \\
Hematocrit & $\mathrm{Ht}(\%)$ & 49 & 40.54 & 3.54 & $29.2-52.3$ \\
White blood cell counts & $\mathrm{WBC} *$ & 49 & 6.04 & 1.65 & $3.1-11.0$ \\
Red blood cell count & $\mathrm{RBC} * *$ & 49 & 4.47 & .40 & $3.83-5.71$ \\
Red cell distribution width & $\mathrm{RDW}(\%)$ & 48 & 12.80 & 1.75 & $10.9-23.3$ \\
Haemoglobin concentration & $\mathrm{Hb}(\mathrm{g} / \mathrm{dl})$ & 49 & 13.31 & 1.12 & $10.0-15.8$ \\
Mean corpuscular haemoglobin & $\mathrm{MCH}(\mathrm{pg})$ & 49 & 29.81 & 1.95 & $24.5-33.6$ \\
Mean corpuscular Hb concentration & $\mathrm{MCHC}(\mathrm{g} / \mathrm{dl})$ & 49 & 32.92 & 2.04 & $29.3-43.2$ \\
Mean corpuscular volume & $\mathrm{MCV}(\mathrm{fl})$ & 49 & 90.70 & 5.51 & $71.5-99.6$ \\
Platelet count & $\mathrm{TROM} *$ & 49 & 270.49 & 114.65 & $73.0-532.0$ \\
Mean platelet volume & $\mathrm{MPV}(\mathrm{fl})$ & 49 & 8.60 & .86 & $7.1-12.6$ \\
Total cholesterol & $\mathrm{TC}(\mathrm{mmol} / \mathrm{L})$ & 86 & 5.48 & .92 & $3.46-7.4$ \\
Triglycerides & $\mathrm{TGs}(\mathrm{mmol} / \mathrm{L})$ & 86 & 1.26 & .52 & $.48-3.01$ \\
High-density lipoprotein & $\mathrm{HDL}(\mathrm{mmol} / \mathrm{L})$ & 86 & 1.70 & .51 & $.54-3.31$ \\
Low-density lipoprotein (calculated) & $\mathrm{LDL}(\mathrm{mmol} / \mathrm{L})$ & 86 & 3.19 & .84 & $1.69-4.83$ \\
\hline
\end{tabular}

Note: ${ }^{*} 10^{\wedge} 3 / \mathrm{uL},{ }^{* *} 10^{\wedge} 6 / \mathrm{uL}$.

port. For RNA isolation, we used $2 \times 9 \mathrm{ml}$ lithium heparin coagulant tubes. Within 1 hour after collection one heparin tube for basal RNA was divided in $3 \times 3 \mathrm{ml}$ aliquots. The aliquots were snap-frozen in acetone with dry ice and stored at $-80^{\circ} \mathrm{C}$ during transport. Dexamethasone (dex) was added to the second heparin tube to a final concentration of $10^{-7}$ $M$ within 1 hour after collection. During transport and for exactly 6 hours, the tube was stored in an insulated box with a constant temperature of $37^{\circ} \mathrm{C}$. After 6 hours' incubation the tube was inverted gently 8-10 times and subsequently divided in $3 \times 3$ $\mathrm{ml}$ aliquots. The aliquots were snap frozen in acetone with dry ice and stored at $-80^{\circ} \mathrm{C}$.

For future coagulation studies, blood was collected in a $4.5 \mathrm{ml}$ sodium citrate tube, which during transport was stored in melting ice $\left(0-2^{\circ} \mathrm{C}\right)$. Blood variables including levels of lipids, apolipoproteins, HbA1c (haemoglobin A1c: an index of average blood glucose levels over a 2- to 3-month period) and CRP (C-reactive protein) were measured in samples collected in a $2 \mathrm{ml}$ EDTA tube, which was stored at room temperature (RT) during transport (see Table 1 for blood picture overview). Urine samples were collected in $2 \times 10 \mathrm{ml}$ vacutainers, and stored in melting ice during car transport.

All samples were transported to the laboratory in Leiden. Average transport time was 4 hours and all samples were centrifuged within 6 hours after collection. The degree of haemolysis was determined by observing the color of the plasma after the centrifugation step.

The EDTA, citrate tube and the urine samples were centrifuged 20 minutes at $2000 \times \mathrm{xg} 4^{\circ} \mathrm{C}$. EDTA and cit- rated plasma was harvested from the buffy coat and red blood cells, aliquoted $(0.5 \mathrm{ml})$, snap-frozen in acetone and dry ice, and stored at $-80^{\circ} \mathrm{C}$. The urine was decanted carefully into new plastic tubes without the debris pellet. The EDTA vacutainers with buffy coat and red cells, and the urine samples were stored at $-20^{\circ} \mathrm{C}$. The heparin vacutainer for lymphocyte isolation was centrifuged for 15 minutes at $1000 \mathrm{xg}$ at $4^{\circ} \mathrm{C}$. After the centrifugation step, heparin plasma was divided into 8 subsamples of $0.5 \mathrm{ml}$, snap-frozen and stored at $-80^{\circ} \mathrm{C}$. For lymphocyte isolation $14 \mathrm{ml}$ phosphate-buffered saline (PBS) was added to the heparin blood, buffycoat and remaining plasma, then the diluted blood was added to $10 \mathrm{ml}$ Ficoll.

This was centrifuged 25 minutes at $800 \mathrm{xg}$ at RT. The lymphocytes were pipetted into a new tube and $45 \mathrm{ml}$ PBS was added. The solution was centrifuged 10 minutes at 250xg RT. This washing step was repeated after discarding the supernatant. The lymphocyte pellet was then taken up into $1 \mathrm{ml}$ RPMI: $20 \%$ Fetal Calf Serum (FCS) $10 \%$ and dimethyl sulfoxide (DMSO). Finally, pellets were frozen and stored overnight at $-80^{\circ} \mathrm{C}$ and then transferred to liquid nitrogen for longterm storage. An overview of blood collection, transport and blood handling is listed in Figure 1.

To test the viability of frozen lymphocyte pellets we transfected 8 samples, originating from 4 individuals, with Epstein-Barr virus B95-8. Cells were centrifuged for 10 minutes at $1200 \mathrm{rpm}$. Supernatant was removed and the cell pellet washed in $10.5 \mathrm{ml}$ RPMI with $1 \%$ FCS. After a second centrifugation of 10 minutes at $1200 \mathrm{rpm} \sim 10^{6}$ cells were suspended in $2 \mathrm{ml}$ RPMI plus $15 \%$ FCS and $1 \mathrm{mM} \mathrm{Na-pyruvate}$ and PenStrep and co-cultured with EBV virus. After 

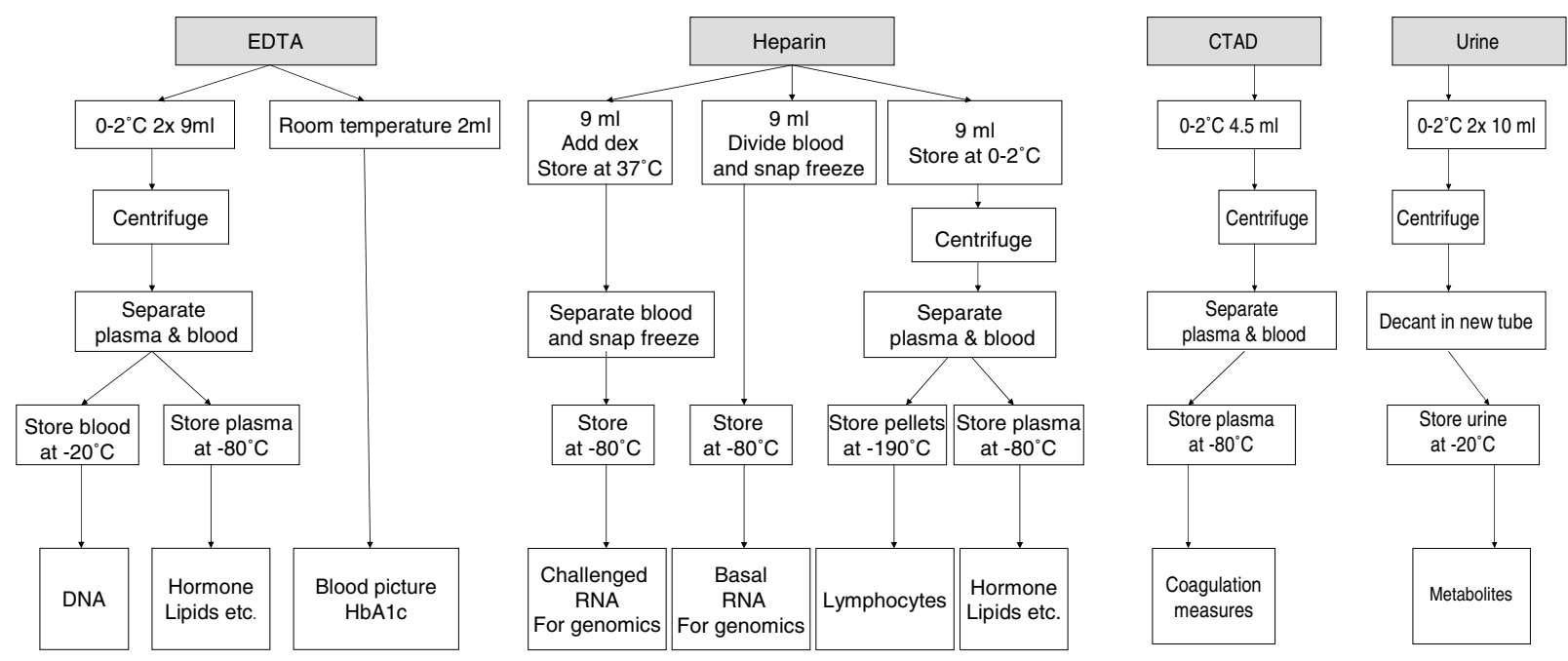

Figure 1

Overview of blood collection and handling, for EDTA, heparin, citrate, and urine tubes.

24 hours the medium with virus was removed and replaced by $2.5 \mathrm{ml}$ RPMI, $15 \%$ FCS $1 \mathrm{mM}$ Na-pyruvate and $1 \mu \mathrm{g} / \mathrm{ml}$ cyclosporine A. This medium was refreshed regularly according to standard procedures.

All samples were handled in the central laboratory in Leiden where labeling of samples and distribution of samples is centralized. The samples obtain a label with double coding: one code represents the individual according to the twin register ID (code of family and position in the family, e.g., father, mother, sister or twin); the other code is sequential and is used to identify the storage position of the sample. The different types of samples are identified by the specific tube or specific cap of the tube or storage box. Labeled isolated samples of DNA are transported to Amsterdam for storage. Labeled cryo-tubes with lymphocytes are sent for storage in liquid nitrogen to Amsterdam. Tubes challenged for gene expression are labeled in addition as A (basal control) and B (challenged) and stored in PAX tubes sequentially in separate boxes in Leiden. Urine is stored in $15 \mathrm{ml}$ tubes sequentially in separate boxes in Leiden. Plasma samples from EDTA blood (purple cap), from heparin blood (green cap), from CTAD blood (blue cap) were stored in boxes containing material from 4 participants in sequential order. The content from each box and the position from each box in trays and the position of each tray in a freezer cache is recorded in an Excel file.

\section{Zygosity Testing}

Twins born to mothers recruited for the study included 22 opposite sex (OS) twin pairs and 24 same-sex (SS) twin pairs. DNA typing from buccal swabs determined information on zygosity of 24 SS twins (Meulenbelt et al., 1995). OS twin pairs were not typed. Zygosity packages were given to the mothers of the twins during the home visit. One SS twin pair was not typed for zygosity because the mother of the twins was missed for blood collection during this pilot study. This twin pair and their mother will participate in the follow-up study. Participants returned the packages by mail. Zygosity testing was performed essentially as described (Rietveld et al., 2000).

\section{$\overline{\text { Results }}$ \\ Participants}

Twenty-three of the 24 MODZT participated in the pilot study. During the telephone interview, one of the MODZT was excluded, because she did not meet the criteria of having a sister with DZ twins. A total of 96 individuals agreed to participate. Eleven participants were not bled (three MODZT, seven parents, one sister). We missed three MODZT who had the third day of their natural cycle at the same time as other participants, due to the large distances between their homes. The parents of the mothers we missed will be visited for blood collection at the same time as their daughters. Three parents of the MODZT were not bled due to health problems. One sister of a MODZT lived in Canada and was therefore not bled. These four participants were asked to participate in DNA collection via buccal swabs. One participant agreed and returned the packages by mail.

In 10 families, two sisters with twins and their parents were bled. In eight families only one of the parents of the ASPs participated (all women), in two of 


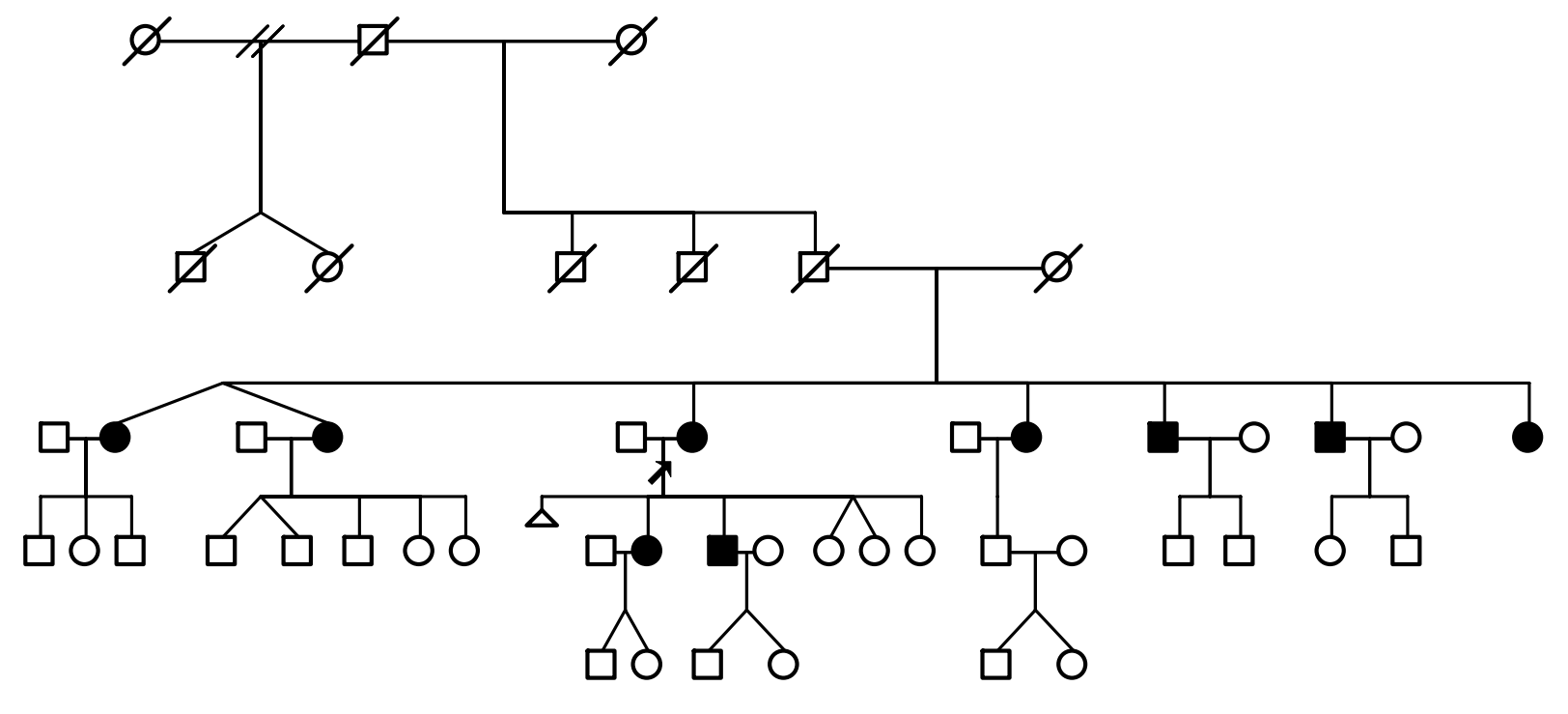

Figure 2

Pedigree of a participating family.

Participating family members are shown with black circles.

them additional siblings participated. In five families both parents were deceased; we therefore asked siblings of the ASPs to participate. A total of 18 additional siblings ( 11 male and seven female) participated. In a few informative and interesting families, other family members were also asked to participate (e.g., grandparents and offspring of the MODZT who had also given birth to DZ twins, see Figure 2).

The mean age of the proband was 44 years (range 28 to 70 years, Table 2) and the mean age of the sister with twins was 46 years (range 25 to 74 years, Table 2). Fourteen women had a natural cycle, 10 used oral contraceptives, 16 were in menopause, and six had had a hysterectomy. Of the proband mothers $39 \%$ had a natural cycle; two of them also had a sister with a natural cycle.

\section{Interview}

All MODZT $(n=46)$ were interviewed by telephone. The average interview time was between 20 and 30 minutes. Table 3 gives an overview of the characteristics of the MODZT obtained from the telephone interview.

Before determination of zygosity by DNA typing from buccal swabs, questions regarding similarity of the twins and experiences mistaking one for another were asked together with the mothers' judgment on the zygosity of the twins. Eighteen of the 24 SS twin pairs were judged by the mother as DZ. Five were judged as probably DZ and one SS twin pair was judged as probably MZ.

The mean age of the twins of the interviewed mothers was 14.5 years. The youngest twin was eight

\section{Table 2}

Mean Age of Proband Mothers and Participating Family Members and Number of Women with Natural Menstrual Cycle, Menopause, Oral Contraceptive (OC) Use or Other.

\begin{tabular}{|c|c|c|c|c|c|c|c|}
\hline \multirow[t]{2}{*}{ Relative } & \multirow[t]{2}{*}{$\mathrm{N}$} & \multirow[t]{2}{*}{ Mean age } & \multirow[t]{2}{*}{ Range } & \multicolumn{4}{|c|}{ Cycle } \\
\hline & & & & Natura & nopause & $O C$ & Other \\
\hline Proband & 23 & 44 & $28-70$ & 9 & 7 & 5 & 2 \\
\hline Sister with twin & 23 & 46 & $25-74$ & 5 & 9 & 5 & 4 \\
\hline Sisters & 7 & 65 & 59-74 & & & & \\
\hline Brothers & 11 & 49 & $37-65$ & & & & \\
\hline Mother & 17 & 69 & $50-80$ & & & & \\
\hline Father & 10 & 68 & $54-78$ & & & & \\
\hline Grandmother with twin & 1 & 80 & & & & & \\
\hline Grandfather & 1 & 82 & & & & & \\
\hline Daughter with twin & 1 & 41 & & & & & \\
\hline Son with twin & 1 & 43 & & & & & \\
\hline Total & 96 & & & & & & \\
\hline
\end{tabular}


Table 3

Interview: Characteristics of Mothers of Dizygotic Twins (Obtained From the Telephone Interview)

\begin{tabular}{lcccc}
\hline & $N$ & Mean & $S D$ & Range \\
\hline Age of mother: conception of twins & 46 & 30.29 & 5.02 & $19-40$ \\
Age of mother: conception first child & 46 & 26.28 & 4.91 & $14-39$ \\
Parity & 46 & 3.33 & 1.19 & $2-7$ \\
Birth weight MODZT(gram) & 38 & 3544 & 553.44 & $2500-5000$ \\
Height (cm) & 46 & 171.33 & 6.56 & $152-185$ \\
Weight (current) & 46 & 69.48 & 6.71 & $55-104$ \\
Minimum weight since 18 years old (kg) & 46 & 59.20 & 14.07 & $45-78$ \\
Maximum weight since 18 years old (kg) & 46 & 74.70 & 14.00 & $55-118$ \\
Weight during twin pregnancy & 42 & 82.38 & 4.14 & $65-119$ \\
BMI (current) & 46 & 23.71 & 3.32 & $17.9-35.6$ \\
Menstrual cycle (days) & 35 & 25.5 & & $19-30$ \\
\hline
\end{tabular}

months old and the oldest nearly 40. The twin mothers gave birth to between two and seven children (mean 3.3). None of the pregnancies was assisted by IVF or a similar treatment. Clomiphene citrate (Clomid) was used to assist a pregnancy in one twin mother, she was one of a sister pair, but not excluded for the pilot study.

Twelve MODZT reported that they had female family members with fertility problems (seven firstdegree family members and five second degree). None of the mothers reported current medical problems.

\section{Transport and Logistics}

All participants $(N=85)$ were visited for blood collection within a period of two months in Dutch wintertime. The average traveling distance was 445 kilometers per day and the average time between collection of the samples and arrival in the laboratory was four hours. We collected blood on the second, third or fourth day of the menstrual cycle in 11 of the 14 women with a natural cycle. We missed 3 women with a natural cycle due to the broad dispersion of these mothers' homes. Participants were awake on average 1.4 hours before the blood collection and they got out of bed between 5.30 a.m. and 8.55 a.m. Two participants were visited before 7.00 a.m., before they went to work, and in four participants we collected blood after 10.00 a.m. (but before 11.00 a.m.) due to traffic jams.

\section{Blood Sampling}

Before blood collection participants were asked the following questions. How is your current physical condition? When was the last time you were ill (fever)? What did you have for breakfast this morning, and did you smoke this morning? We also assessed height and weight. Five of the 85 participants reported that they smoked before blood collection, and seven participants reported not to have fasted.

Sixty-two participants reported that their physical condition was good. Ten participants had a cold, 11 said they did not feel well (no fever) and two were really ill (fever). C-Reactive protein (CRP) was measured as an inflammatory marker in 73 participants. Figure 3 shows the difference in mean CRP levels and reported physical health, divided in two categories, normal health and discomfort/illness. Of the 53 individuals reporting normal health (score 1), only one person showed an increased CRP level above $10 \mathrm{mg} / \mathrm{l}$. Among persons reporting some kind of discomfort or illness (score 2-4), 6 of the 20 participants showed a CRP level above $10 \mathrm{mg} / \mathrm{l}$. We concluded that the questionnaire is mainly effective in excluding acute phase reactions.

Evaluation of the platelet product beta-Thromboglobulin in citrated plasma revealed a value generally above $150 \mathrm{IU} / \mathrm{ml}$ which is well above the expected 50 $\mathrm{IU} / \mathrm{ml}$ and indicative of release of platelet products or activation of platelets during transport and handling (Kluft \& Meijer, 1996). This precludes assay of some haemostatic variables including PAI-1. To reduce this effect in the future we will use CTAD anticoagulant mixture, which exerts platelet stabilization (Kluft et al., 1996).

\section{$\overline{\text { Discussion }}$}

This pilot study has shown that, in the Netherlands, it is feasible to collect blood and urine samples for research purposes by making home visits to participants. We collected samples in nuclear families in the morning after overnight fasting. After the home visit, blood samples were immediately transported by car to the laboratory. Women with a natural cycle were visited on days two to four of their menstrual cycle and women on oral contraceptives were visited during their pill-free week. In this pilot study both the proband mother and her sister were interviewed; in future research all mothers of DZ twins will participate in the telephone interview.

Lymphocytes and RNA can be extracted with sufficient yield and quality. For lymphocytes blood was frozen at $-80^{\circ} \mathrm{C}$ during transport and then transferred to liquid nitrogen for long-term storage. For RNA blood was frozen at $-80^{\circ} \mathrm{C}$ and for a maximum of one 


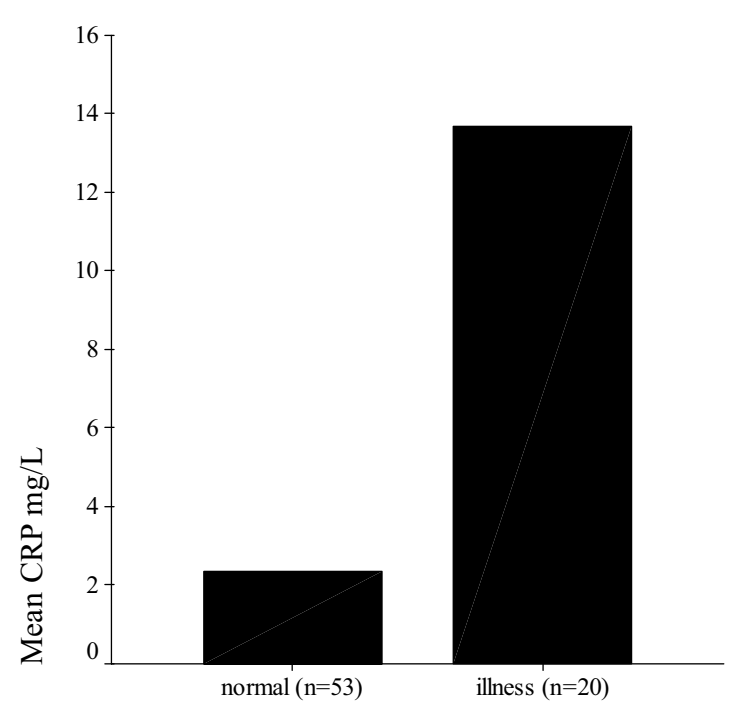

Reported physical health

\section{Figure 3}

Reported physical condition and CRP-level $(N=85)$.

month. RNA isolated from whole blood showed no degradation of transcripts and it should be feasible to perform gene-expression profiling studies with these samples. The effects of prolonged storage ( $>1$ month) on the amount of extracted RNA in polyethylene aliquots are currently not known (see accompanying paper, Spijker et al., 2004). In future research, we may also challenge blood cells with immune systems related lipopolysaccharide (LPS), since greater shifts in gene-expression were observed during our pilot study than after a dexamethasone challenge.

To obtain representative plasma variables, the materials have to be stable for all individuals. We performed our pilot in the winter period with a significant number of flu cases in the Netherlands. We observed an increased CRP > $10 \mathrm{mg} / \mathrm{l}$ in 7 individuals raising the question whether some plasma variables for these individuals are in a stable phase. The presence of high CRP indicates an acute phase reaction, which may influence multiple quantitative traits and affect the analysis. We observed that a questionnaire is insufficient to predict high CRP, but excellent in predicting $\mathrm{CRP}<10 \mathrm{mg} / \mathrm{l}$. In future, we have to decide whether we will exclude cases with CRP $>10$ $\mathrm{mg} / \mathrm{l}$ or resample. The number of high values is expected to be less in other periods of the year.

In future research we will modify the sampling of citrate tubes, by using CTAD tubes in an attempt to stabilize the platelets better. In a preliminary study with lithium heparin we observed that an interaction with platelets occurs that dictates we first prepare platelet-rich plasma and separate white cells from platelets. This is not required for Natrium heparin and would reduce the complexity of the procedures.
However, the lymphocyte harvest is better with lithium heparin.

This study focused on families with affected sister pairs (both sisters are mothers of DZ twins). It is a pilot project for a larger study which aims to localize and identify the genes causing variation in human DZ twinning. Families from the Netherlands, Australia and New Zealand participate in the study. Outside the Netherlands participants are bled by their local general practitioner and the samples are returned with courier service to the Queensland Institute of Medical Research. This procedure is an efficient and cheap way of collecting DNA samples, but limited, when blood samples need special handling after collecting for RNA, lymphocytes and other blood parameters. Another advantage of our approach is that transport and blood handling, with a special team of motivated trained nurses and analysts will minimize error and ensure high quality of the blood samples.

Of the 96 participants, 85 were bled. We could not visit 11 participants, because distances between these participants' homes were too great to be feasible to cover in one day. This was a relatively small pilot group and we do not expect these logistic problems with larger study designs. Home visits for blood and urine collection resulted in high response rates and cooperation. We expect that cooperation and response rates will decline when participants have to be active in providing blood samples (e.g., leaving their homes), especially when participants are older (e.g., the parents of the affected sister pairs). We will therefore use this sample protocol in studies of other complex traits. The protocol is currently implemented in the genetic study of migraine, cardiovascular disease of attention and memory, and of depression. These projects, including the twinning study, will approach twin families registered with Netherlands Twin Register. Initially we will invite 8000 participants. In this feasibility study, we focused on twinning because it presented the most complex logistics. The nationwide standardized blood sampling and laboratory organization makes it possible to create a large, informative biobank.

In combination with the advances in molecular technologies, such a biobank will help to broaden the knowledge of the endocrinology and of the genes involved in human DZ twinning and other complex traits. In the study of DZ twinning hormone concentrations of, for example, LH, FSH, inhibin A and B will be determined so that genome scans can be conducted for linkage with endocrine parameters. Likewise, once the genome-wide markers become available, other parameters, which have been assessed during the interview and home visit (e.g., height and weight) and in plasma (e.g., lipids and apolipoproteins) can be used for sib-pair based linkage analyses.

In conclusion, the pilot study showed that nationwide sample collection is feasible in Dutch families with two or more sisters who have given birth to spontaneous dizygotic twins. Large-scale blood and 
urine sample collection for this project will be combined with similar sample collection in several other linkage projects and will start in the fall of 2004.

In July 2004, we started recruiting families for the proposed DZ twinning study. Of the families approached around $70 \%$ agreed to participate. Sample collection, transport, blood handling and laboratory organization will be based and standardized on the results from this pilot study.

\section{Acknowledgments}

We gratefully acknowledge the financial support from the National Institute of Child Health and Human Development (Grant number HD042157) and the GenomeUTwin project, which is supported by the European Union Contract No. QLG2-CT-2002-01254. The study was done within the Centre for Neurogenomics and Cognition Research (CNCR), the Centre for Medical Systems Biology, a centre of excellence supported by the Netherlands Genomics Initiative/ Netherlands Organisation for Scientific Research (NWO).

\section{$\overline{\text { References }}$}

Austin, M. A., Harding, S., \& McElroy, C. (2003). Genebanks: A comparison of eight proposed international genetic databases. Community Genetics, 6, 37-45.

Boomsma, D. I., Frants, R. R., Bank, R. A., \& Martin, N. G. (1992). Protease inhibitor (Pi) locus, fertility and twinning. Human Genetics, 89, 329-332.

Bulmer, M. G. (1970). The biology of twinning in man. Oxford, UK: Clarendon Press.

Galloway, S. M., McNatty, K. P., Cambridge, L. M., Laitinen, M. P., Juengel, J. L., Jokiranta, T. S., McLaren, R. J., Luiro, K., Dodds, K. G., Montgomery, G. W., Beattie, A. E., Davis, G. H., \& Ritvos, O. (2000). Mutations in an oocyte-derived growth factor gene (BMP15) cause increased ovulation rate and infertility in a dosage-sensitive manner. Nature Genetics, 25, 279-283.

Hanrahan, J. P., Gregan, S. M., Mulsant, P., Mullen, M., Davis, G. H., Powell, R., \& Galloway, S. M. (2004). Mutations in the genes for oocyte-derived growth factors GDF9 and BMP15 are associated with both increased ovulation rate and sterility in Cambridge and Belclare sheep (Ovis aries). Biology of Reproduction, 70, 900-909.

Kaiser, J. (2002). Biobanks: Population databases boom, from Iceland to the U.S. Science, 298, 1158-1161.

Kluft, C., \& Meijer, P. (1996). Update 1996: Blood collection and handling procedures for assessment of plasminogen activators and inhibitors (Leiden Fibrinolysis Workshop). Fibrinolysis, 10, 171-179.

Lambalk, C. B., Boomsma, D. I., De Boer, L., De Koning, C. H., Schoute, E., Popp-Snijders, C., \& Schoemaker, J.
(1998). Increased levels and pulsatility of follicle-stimulating hormone in mothers of hereditary dizygotic twins. The Journal of Clinical Endocrinology and Metabolism, $83,481-486$.

Lewis, C. M., Healey, S. C., \& Martin, N. G. (1996). Genetic contribution to DZ twinning. American Journal of Medical Genetics, 61, 237-246.

Martin, N. G., Olsen, M. E., Theile, H., El Beaini, J. L., Handelsman, D., \& Bhatnagar, A. S. (1984). Pituitaryovarian function in mothers who have had two sets of dizygotic twins. Fertility and Sterility, 41, 878-880.

Martin, N. G., Robertson, D. M., Chenevix-Trench, G., de Kretser, D. M., Osborne, J., \& Burger, H. G. (1991). Elevation of follicular phase inhibin and luteinizing hormone levels in mothers of dizygotic twins suggests nonovarian control of human multiple ovulation. Fertility and Sterility, 56, 469-474.

Meulemans, W. J., Lewis, C. M., Boomsma, D. I., Derom, C. A., Van den Berghe, H., Orlebeke, J. F., Vlietinck, R. F., \& Derom, R. M. (1996). Genetic modelling of dizygotic twinning in pedigrees of spontaneous dizygotic twins. American Journal of Medical Genetics, 61, 258-263.

Meulenbelt, I., Droog, S., Trommelen, G. J., Boomsma, D. I., \& Slagboom, P. E. (1995). High-yield noninvasive human genomic DNA isolation method for genetic studies in geographically dispersed families and populations. American Journal of Human Genetics, 57, 1252-1254.

Montgomery, G. W., Zhao, Z. Z., Morley, K. I., Marsh, A. J., Boomsma, D. I., Martin, N. G., \& Duffy, D. L. (2003). Dizygotic twinning is not associated with methylenetetrahydrofolate reductase haplotypes. Human Reproduction, 18, 2460-2464.

Nederhand, R. J, Droog, S., Kluft, C., Simoons, M. L., \& de Maat, M. P. M. (2003). Logistics and quality control for DNA sampling in large multicenter studies. Journal of Thrombosis and Haemostasis, 1, 987-991.

Rietveld, M. J., Der Valk, J. C., Bongers, I. L., Stroet, T. M., Slagboom, P. E., \& Boomsma, D. I. (2000). Zygosity diagnosis in young twins by parental report. Twin Research, 3, 134-141.

Spijker, S., van de Leemput, J. C. H., Hoekstra, C., Boomsma, D. I., \& Smit, A. B. (2004). Profiling gene expression in whole blood samples following an in vitro challenge. Twin Research, 7, 564-570.

Wilson, T., Wu, X. Y., Juengel, J. L., Ross, I. K., Lumsden, J. M., Lord, E. A., Dodds, K. G., Walling, G. A, McEwan, J. C., O'Connell, A. R., McNatty, K. P., Montgomery, G. W. (2001). Highly prolific Booroola sheep have a mutation in the intracellular kinase domain of bone morphogenetic protein IB receptor (ALK-6) that is expressed in both oocytes and granulosa cells. Biology of Reproduction, 64, 1225-1235. 